\title{
Downregulation of NAD(P)H:quinone oxidoreductase 1 inhibits proliferation, cell cycle and migration of cholangiocarcinoma cells
}

\author{
SIRIWOOT BUTSRI $^{1}$, VEERAPOL KUKONGVIRIYAPAN ${ }^{1}$, LADDAWAN SENGGUNPRAI ${ }^{1}$, \\ SARINYA KONGPETCH $^{1}$, PONSILP ZEEKPUDSA ${ }^{2}$ and AUEMDUAN PRAWAN ${ }^{1}$ \\ ${ }^{1}$ Department of Pharmacology, Faculty of Medicine, Liver Fluke and Cholangiocarcinoma Research Center, \\ Khon Kaen University, Khon Kaen 40002; ${ }^{2}$ Chulabhorn International College of Medicine, \\ Thammasat University, Pathum Thani 12120, Thailand
}

Received August 10, 2015; Accepted February 13, 2017

DOI: $10.3892 / 01.2017 .5951$

\begin{abstract}
We previously reported that upregulation of $\mathrm{NAD}(\mathrm{P}) \mathrm{H}:$ quinone oxidoreductase 1 (NQO1) in cholangiocarcinoma (CCA; a fatal bile duct cancer) was associated with poor prognosis. It was also demonstrated that the suppression of NQO1 was able to enhance the chemosensitivity of CCA cells. In the present study, in order to elucidate the biological role of NQO1 in CCA, the effects of small interfering RNA (siRNA)-mediated knockdown of NQO1 on cell proliferation, cell cycle and migration were determined in KKU-100 CCA cells, which notably expressed NQO1. The cell proliferation ability and cell cycle distribution were identified by clonogenic cell survival assay and flow cytometric analysis, respectively. Wound healing and Transwell migration assays were performed to evaluate cell migration. The molecules involved in cell proliferation and migration were determined by western blot analysis and reverse transcription-quantitative polymerase chain reaction analysis. The results demonstrated that NQO1 siRNA-mediated knockdown effectively impaired colony formation capacity, induced cell cycle arrest at the $\mathrm{G}_{1}$ phase and suppressed migration of KKU-100 cells. CCA cells transfected with NQO1 siRNA exhibited increased expression levels of p21 and decreased cyclin D1 protein expression levels. Furthermore, the ratio of matrix metalloproteinase 9/tissue inhibitors of metalloproteinases 1 (TIMP1) mRNA expression level was decreased in the NQO1-knockdown cells. Therefore, the present study provided evidence supporting the biological role of NQO1 in the regulation of cell proliferation, cell cycle and migration of CCA cells. Therefore, NQO1 may prove to be a potential molecular target to enhance CCA treatment.
\end{abstract}

Correspondence to: Dr Auemduan Prawan, Department of Pharmacology, Faculty of Medicine, Liver Fluke and Cholangiocarcinoma Research Center, Khon Kaen University, 123 Mittraparp Highway, Muang District, Khon Kaen 40002, Thailand

E-mail: peuamd@kku.ac.th

Key words: $\mathrm{NAD}(\mathrm{P}) \mathrm{H}: q u i n o n e$ oxidoreductase 1, cholangiocarcinoma, cell proliferation, cell cycle, migration

\section{Introduction}

Cholangiocarcinoma (CCA; a fatal bile duct cancer) is a major public health concern in the Lower Mekong Basin and South East Asia, which are areas endemic for Opisthorchis viverrini liver fluke infection (1). The prognosis of CCA is principally poor because the majority of patients with CCA are diagnosed at an advanced stage, therefore they are inoperable and there are no effective treatments available (2). Additionally, CCA is prone to developing multidrug chemoresistance $(3,4)$. Therefore, there is a requirement to investigate novel targeted therapies and strategies to enhance chemosensitivity of CCA.

We previously demonstrated that the alteration of cytoprotective enzymes or derangement of intracellular redox balance and the signaling system were involved in the chemoresistance of CCA (5-8). NAD(P)H:quinone oxidoreductase 1 (NQO1; EC 1.6.5.2), one of the detoxifying enzymes with antioxidant properties, has been proposed to be associated with the chemotherapeutic response of CCA $(5,8)$. NQO1 is generally recognized as a 'cell protector', its induction in response to various noxious stimuli provides protection for cells against oxidative damage and oxidative stress-associated pathological conditions including cancer $(9,10)$. Conversely, an increasing number of studies revealed abnormal increases in NQO1 expression levels in solid tumors of the adrenal gland, breast, colon, lung, ovary, pancreas, thyroid, skin and bladder (9-16). High-level expression of NQO1 may be associated with cancer progression and it was suggested to be a poor prognostic marker of these types of cancer $(14,16,17)$. Upregulation of NQO1 during carcinogenesis may provide cancer cells with a growth advantage and protection against extreme oxidative stress environments $(10,11)$. Considering the function of NQO1, an increased NQO1 expression level may be associated with disappointing outcomes to certain cancer treatment modalities, including chemotherapy and radiotherapy, which induces cancer cell death by the generation of free radicals and oxidative damage $(5,8)$.

The roles of NQO1 during carcinogenesis and chemotherapeutic response have been demonstrated by numerous previous studies $(11,18,19)$. Inhibition of NQO1 by a pharmacological inhibitor, dicoumarol, suppressed urogenital and pancreatic cancer cell growth and also potentiated cytotoxicity of 
cisplatin and doxorubicin $(18,20)$. Similarly, the roles of NQO1 in CCA have been previously demonstrated $(5,8,17,21)$. Significant association between high NQO1 expression level in CCA tissues and short survival time of patients was observed (17), implying NQO1 is an independent predictor associated with prognosis of CCA. Furthermore, dicoumarol was able to enhance gemcitabine-induced cytotoxicity in CCA cells with increased NQO1 activity (5). In addition, knockdown of NQO1 expression levels enhanced the cytotoxicity of chemotherapeutic agents; conversely, overexpression of NQO1 protected the cells from chemotherapeutic agents (8). These results suggested roles for NQO1 in CCA chemotherapy; however, the biological role of NQO1 in CCA cells has not yet been clearly demonstrated.

The aim of the present study was to investigate the biological role of NQO1 in CCA cells. The effects of NQO1 knockdown on cell proliferation, cell cycle and migration were assessed in KKU-100 CCA cells, which notably expressed NQO1. Furthermore, the molecular events associated with NQO1 small interfering RNA (siRNA)-induced inhibition of cell proliferation, inducing cell cycle arrest and inhibiting migration of CCA cells were investigated.

\section{Materials and methods}

Human cell line and cell culture. KKU-100 cells with high expression levels of NQO1 were provided by Professor Banchob Sripa (Department of Pathology, Faculty of Medicine, Khon Kaen University, Khon Kaen, Thailand). KKU-100 cells were established, characterized and derived from CCA tissues (22). Cells were routinely cultured in Ham's F-12 complete medium (Gibco; Thermo Fisher Scientific, Inc., Waltham, MA, USA) supplemented with $10 \%$ fetal bovine serum (Gibco; Thermo Fisher Scientific, Inc.), $10 \mathrm{mM}$ 4-(2-hydroxyethyl)-1-piperazineethanesulfonic acid ( $\mathrm{pH}$ 7.3), $100 \mathrm{U} / \mathrm{ml}$ penicillin $\mathrm{G}$ and $100 \mu \mathrm{g} / \mathrm{ml}$ streptomycin, and maintained under an atmosphere of $5 \% \mathrm{CO}_{2}$ at $37^{\circ} \mathrm{C}$. Cells were passaged every 3 days using $0.25 \%$ trypsin-EDTA (2).

NQO1 siRNA transfection. The transfection of NQO1 siRNA was performed using four sequences of predesigned NQO1 siRNA (siGENOME SMARTpool of siRNA M-005133-02-0010; Dharmacon Inc., Lafayette, CO, USA), as described previously (8). The NQO1 siRNA and the negative control siRNA (siGENOME non-targeting siRNA pool\#2 D-001206-14-20) at a final concentration of $100 \mathrm{nM}$ siRNA were introduced to the cells using Lipofectamine ${ }^{\mathrm{TM}} 2000$ (Invitrogen; Thermo Fisher Scientific, Inc.) as a transfection reagent, according to the manufacturer's protocol. The efficiency of the NQO1 knockdown by transient NQO1 siRNA transfection was confirmed by evaluating levels of gene expression by performing the reverse transcription-quantitative polymerase chain reaction (RT-qPCR), protein expression levels by performing western blot analysis and NQO1 enzyme activity using an enzymatic assay.

Clonogenic survival assay. Cells with NQO1 siRNA were used in the colony formation assay. KKU-100 cells were transfected with NQO1 siRNA then seeded in 6-well plates at densities of 300 and 600 cells/well in Ham's F-12 complete medium.
Two cell densities were used to confirm the effect of $\mathrm{NQO}_{1}$ siRNA on replicative potential of KKU-100 cells. Cells were cultured at $37^{\circ} \mathrm{C}$ in a $5 \% \mathrm{CO}_{2}$ incubator for another 10 days to obtain cell colonies and the Ham's F-12 complete medium was freshly renewed every 3 days. On the last experiment day, the cell colonies were fixed with absolute methanol at room temperature $\left(25^{\circ} \mathrm{C}\right)$ for $20 \mathrm{~min}$, stained with $0.5 \%$ crystal violet solution at room temperature $\left(25^{\circ} \mathrm{C}\right)$ for $15 \mathrm{~min}$ and the number of cell colonies was counted (23).

Cell cycle analysis using flow cytometry. KKU-100 cells were seeded in a 6-well plate with a density of 150,000 cells/well in Ham's F-12 complete medium, transfected with NQO1 siRNA for $24 \mathrm{~h}$ and then starved for $12 \mathrm{~h}$ using serum-free Ham F-12 medium at $37^{\circ} \mathrm{C}$ and $5 \% \mathrm{CO}_{2}$. At $24 \mathrm{~h}$ post-starvation, the cell cycle distribution was evaluated using flow cytometric analysis. On the experimental day, cells were harvested, washed with PBS and fixed overnight with $70 \%$ cold ethanol at $4^{\circ} \mathrm{C}$. Subsequently, the cell suspension was maintained at $-20^{\circ} \mathrm{C}$ for $3 \mathrm{~h}$, followed by the addition of $0.02 \mathrm{mg} / \mathrm{ml}$ propidium iodide and $0.02 \mathrm{mg} / \mathrm{ml}$ RNase A (Ameresco, Inc., Framingham, MA, USA). The cell suspension was incubated further for $1 \mathrm{~h}$ at $4^{\circ} \mathrm{C}$ in the dark. The cell cycle stage content was evaluated using a BD FACSCanto II flow cytometer and determined using BD FACSDiva $^{\mathrm{TM}}$ software v6.1.3 (BD Biosciences, San Jose, CA, USA). The service was provided by the Research Instrument Center, Khon Kaen University, Thailand.

Cell motility by wound healing and Transwell migration assays. Cells with NQO1 siRNA were used in the wound-healing and Transwell migration assays. For the wound-healing assay, KKU-100 cells transfected with NQO1 siRNA were seeded in a 24-well plate at a cell density of 150,000 cells/well in Ham's F-12 complete medium at $37^{\circ} \mathrm{C}$ with $5 \% \mathrm{CO}_{2}$ overnight to obtain $90-100 \%$ cell confluence. On the next day, cells were scratched to create a straight wound using a sterile $200-\mu 1$ pipette tip. Following scratching, the cells were washed twice with PBS to remove any detached cells and further incubated for $72 \mathrm{~h}$ at $37^{\circ} \mathrm{C}$ and $5 \% \mathrm{CO}_{2}$. The width of the wound outline was monitored under a phase-contrast microscope at designed times; $0,3,6,12,24,48$ and $72 \mathrm{~h}$.

For the Transwell migration assay, KKU-100 cells transfected with NQO1 siRNA were seeded onto a $6.5 \mathrm{~mm}$ Transwell insert (Corning Incorporated, Corning, NY, USA) at a cell density of 75,000 cells/well in serum-free Ham's F-12 medium at $37^{\circ} \mathrm{C}$ with $5 \% \mathrm{CO}_{2}$, while the lower parts of the chambers were filled with Ham's F-12 complete medium. Following a 24-h incubation, cells were fixed with absolute methanol for $20 \mathrm{~min}$ and stained with $0.05 \%$ crystal violet solution for $15 \mathrm{~min}$. The number of migrated cells on the lower surface of the filters was counted under a light microscope (Eclipse Ni-U; Nikon Corporation, Tokyo, Japan).

Western blot analysis. In order to determine the expression levels of p21, cyclin D1 and cyclin A protein, western blot analysis was performed. KKU-100 cells were seeded in a 6-well plate at a cell density of 150,000 cells/well in Ham's F-12 complete medium and transfected with NQO1 siRNA for $24 \mathrm{~h}$. Then cells were serum-starved for $12 \mathrm{~h}$ using serum-free Ham F-12 medium at $37^{\circ} \mathrm{C}$ in at atmosphere containing 5\% 
$\mathrm{CO}_{2}$ and grown in Ham's F-12 complete medium for a further $24 \mathrm{~h}$. Following this, the cells were harvested for western blot analysis. Cells were washed twice with PBS and lysed with radioimmunoprecipitation assay cell lysis buffer supplemented with dithiothreitol, phenylmethylsulfonyl fluoride (PMSF) and protease inhibitor cocktail (Ameresco, Inc.) at $4^{\circ} \mathrm{C}$ for $15 \mathrm{~min}$. The concentration of extracted proteins was determined using Bradford reagent, according to the manufacturer's protocol (Bio-Rad Laboratories, Inc., Hercules, CA, USA). Whole cell lysate containing $30 \mathrm{mg}$ protein was separated by SDS-PAGE using a $10 \%$ polyacrylamide gel. The separated proteins were then transferred onto polyvinylidene difluoride membranes (EMD Millipore, Billerica, MA, USA). The membranes were blocked for $1 \mathrm{~h}$ at room temperature with $5 \%$ non-fat milk in PBS supplemented with $0.1 \%$ Tween-20. They were then probed with rabbit polyclonal anti-human cyclin D1 (dilution, 1:1,000; \#sc-718), rabbit polyclonal anti-human cyclin A (dilution, 1:2,000; \#sc-751), mouse monoclonal anti-human p21 (dilution, 1:500; \#sc-56335) or horseradish peroxidase (HRP)-conjugated goat polyclonal anti-human $\beta$-actin (dilution, 1:5,000; \#sc-1616) primary antibodies for $12 \mathrm{~h}$ at $4^{\circ} \mathrm{C}$ (all antibodies were from Santa Cruz Biotechnology, Inc., Dallas, TX, USA). The membranes were then incubated with HRP-conjugated goat anti-mouse IgG (dilution, 1:5,000; \#sc-2005) or HRP-conjugated goat anti-rabbit IgG (dilution, 1:5,000; \#sc-2004) secondary antibodies for $2 \mathrm{~h}$ at room temperature (both from Santa Cruz Biotechnology Inc.). The membranes were incubated with enhanced chemiluminescence (ECL) substrate solution (Amersham ${ }^{\mathrm{TM}} \mathrm{ECL}^{\mathrm{TM}}$ Prime Western Blotting detection reagent; GE Healthcare Life Sciences, Chalfont, UK) for $1 \mathrm{~min}$ at room temperature $\left(25^{\circ} \mathrm{C}\right)$. The optical densities of the protein bands were determined using ImageQuant ${ }^{\mathrm{TM}}$ LAS4000 (GE Healthcare Life Sciences) (8).

$R T-q P C R$. In order to determine the mRNA expression levels of matrix metalloproteinases (MMPs) and tissue inhibitors of metalloproteinases (TIMPs), RT-qPCR was performed. KKU-100 cells were seeded in a 6-well plate with density of 150,000 cells/well in Ham's F-12 complete medium, transfected with NQO1 siRNA for $24 \mathrm{~h}$ and then starved for $12 \mathrm{~h}$ using serum-free Ham F-12 medium at $37^{\circ} \mathrm{C}$ and $5 \%$ $\mathrm{CO}_{2}$. At $24 \mathrm{~h}$ post-starvation, total RNA was extracted from CCA cells using TRIzol ${ }^{\boxplus}$ reagent was obtained from Thermo Fisher Scientific, Inc., according to a previously described method (8). A cDNA synthesis mixture consisting of $1 \mu \mathrm{g}$ total RNA and $4 \mu 1$ 5x iScript ${ }^{\mathrm{TM}}$ Reverse Transcription Supermix for RT-qPCR (170-8841; Bio-Rad Laboratories, Inc.) was mixed with RNase-free water in a total volume of $20 \mu 1$. cDNA synthesis was performed using a $\mathrm{C} 1000^{\mathrm{TM}}$ thermal cycler (Bio-Rad Laboratories, Inc.). The cDNA synthesis conditions included priming for $5 \mathrm{~min}$ at $25^{\circ} \mathrm{C}$ and reverse transcription for $30 \mathrm{~min}$ at $42^{\circ} \mathrm{C}$, and the reaction was stopped by incubation for $15 \mathrm{~min}$ at $70^{\circ} \mathrm{C}$. The reverse transcription products served as templates for qPCR. PCR amplification was performed using specific primers for the MMP2, MMP3, MMP9, TIMP1, TIMP2 and the internal control $\beta$-actin. The primer sequences were as follows: i) MMP2 (NM_004530) (24), forward primer 5'-AGCTCCCGGAAA AGATGGATG-3' and reverse primer 5'-CAGGGTGCTGGC TGAGTAGAT-3'; ii) MMP3 (NM_002422) (25), forward primer 5'-GCGTGGATGCCGCATATGAAGTTA-3' and reverse primer 5'-AAACCTAGGGTGTGGATGCCTCTT-3'; iii) MMP9 (NM_002422) (26), forward primer 5'-GAAGAT GCTGCTGTTCAGCG-3' and reverse primer 5'-ACTTGG TCCACCTGGTTCAA-3'; iv) TIMP1 (NM_003254) (27), forward primer 5'-AGGCTCTGAAAAGGGCTTCCA-3' and reverse primer 5'-GAGTGGGAACAGGGTGGACA-3'; v) TIMP2 (NM_003255) (28), forward primer 5'-GACGGC AAGATGCACATCAC-3' and reverse primer 5'-GAGATG TAGCACGGGATCATGG-3'; vi) $\beta$-actin (NM_001101) (8), forward primer 5'-TGCCATCCTAAAAGCCAC-3' and reverse primer 5'-TCAACTGGTCTCAAGTCAGTG-3'. The reverse-transcription fluorescence PCR, based on EvaGreen ${ }^{\circledR}$ dye, was performed in a final volume of $20 \mu 1$ of $1 \mathrm{x}$ SsoFast ${ }^{\mathrm{TM}}$ EvaGreen ${ }^{\circledR}$ Supermix (172-5201; Bio-Rad Laboratories, Inc.), $0.5 \mu \mathrm{mol} / 1$ MMP2, MMP3, MMP9, TIMP1 or TIMP2 primer and $0.25 \mu \mathrm{mol} / 1 \beta$-actin primer. A negative control (no cDNA template) was included in the experimental runs. The qPCR was performed for each gene in duplicate on cDNA samples in 96-well reaction plates using LightCycler ${ }^{\circledR} 480$ Real-Time PCR System (Roche Applied Science, Madison, WI, USA). The cycling conditions for qPCR were: $95^{\circ} \mathrm{C}$ for $3 \mathrm{~min}$, followed by 40 cycles of $95^{\circ} \mathrm{C}$ for $15 \mathrm{sec}$ and $60^{\circ} \mathrm{C}$ for $31 \mathrm{sec}$. To verify the purity of the products, a melting curve analysis was performed following each run. The concentration of PCR products was evaluated on the basis of an established standard curve derived from serial dilutions of the positive control for MMP2, MMP3, MMP9, TIMP1, TIMP2 and $\beta$-actin.

Statistical analysis. Data were expressed as the mean \pm standard error of the mean from three independent experiments (SigmaPlot Version 10.0; Systat Software, Inc., San Jose, CA, USA). Statistical comparison between the control and treatment groups was performed using Student's t-test. $\mathrm{P}<0.05$ was considered to indicate a statistically significant difference (SigmaStat Version 3.11; Systat Software, Inc.).

\section{Results}

Knockdown of NQO1 expression suppresses the cell proliferation of KKU-100 cells. The efficiency of NQO1 siRNA transfection in KKU-100 cells was established using qPCR, western blot analysis and NQO1 enzymatic assay (Fig. 1). Knockdown of NQO1 transcripts using siRNA transfection efficiently decreased NQO1 expression at the mRNA and protein levels, and enzymatic activity by $\sim 90, \sim 60$ and $\sim 50 \%$, respectively compared with the non-targeting siRNA-transfected control cells $(\mathrm{P}<0.001$; Fig. 1$)$. To investigate the role of NQO1 on CCA cell proliferation, NQO1-knockdown cells using siRNA were subjected to the clonogenic survival assay. The colony formation, as an index of long-term cell proliferation, was observed by plating the cells at low densities (300 and 600 cells/well; Fig. 2). NQO1-knockdown CCA cells exhibited a decreased number of colonies formed compared with control cells (Fig. 2). A significant antiproliferative effect of NQO1 siRNA was observed between days 6 and 10. At 10 days of culture, the NQO1 siRNA decreased colony formation by $\sim 24$ and $\sim 37 \%$ when cells were plated at 300 and 600 cells/well, respectively, compared with the 
$\mathbf{A}$
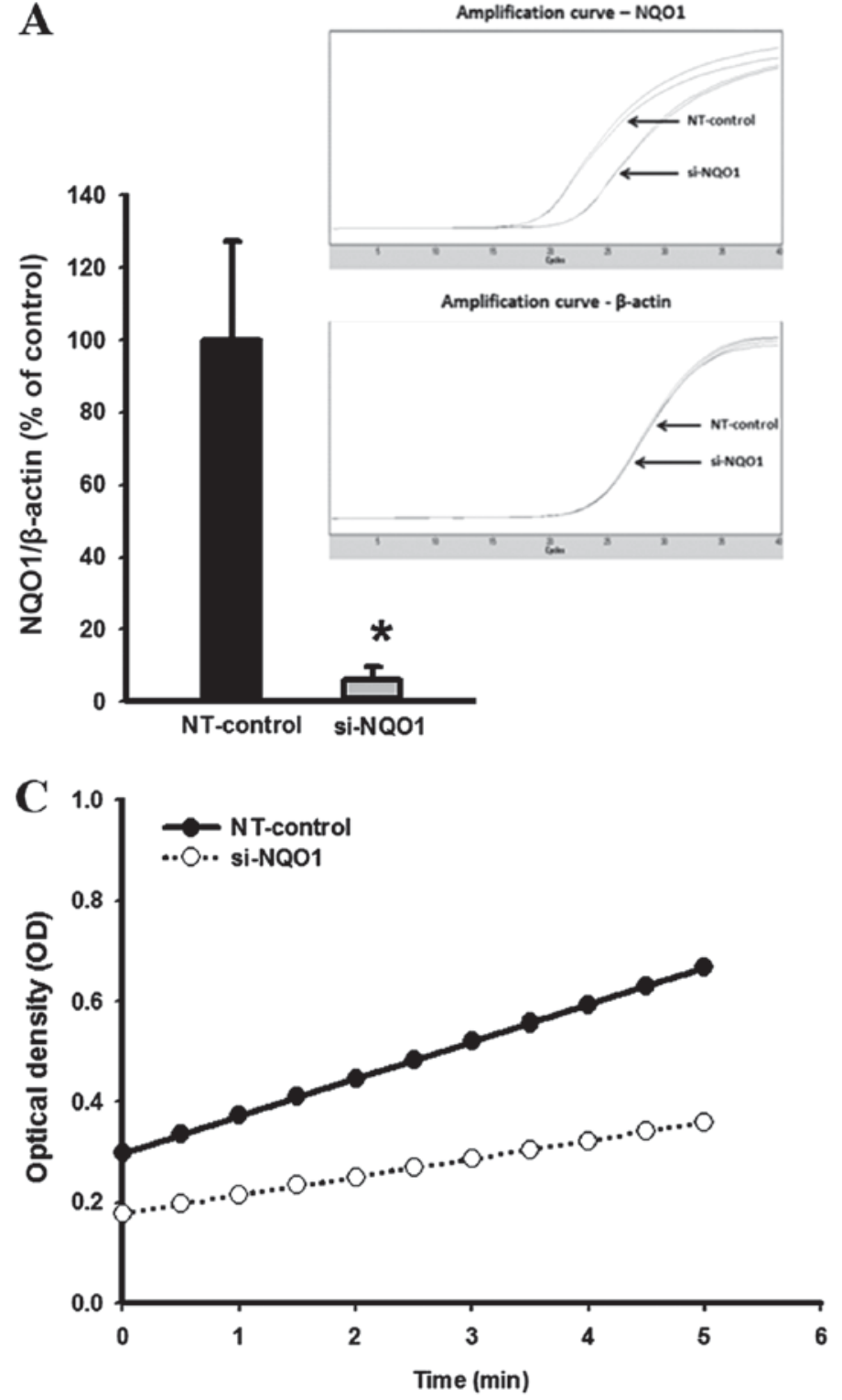

B
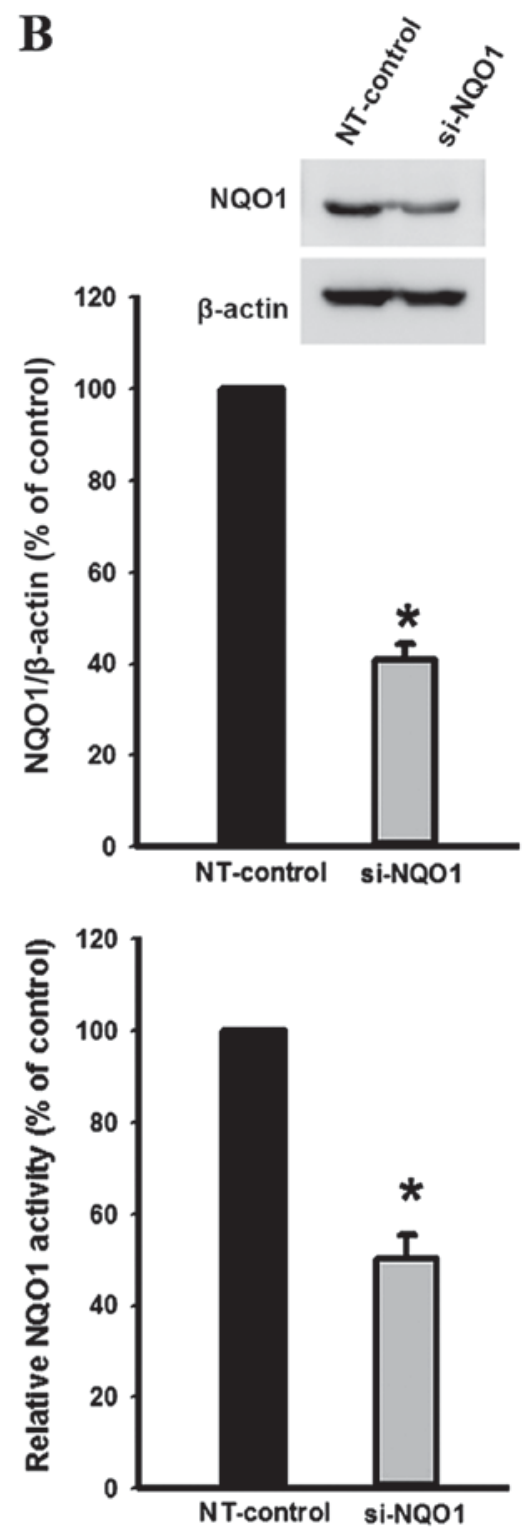

Figure 1. NQO1 siRNA was effectively transfected in KKU-100 cells. NQO1-knockdown cells using siRNA were used to assess levels of (A) NQO1 mRNA, (B) NQO1 protein and (C) NQO1 enzyme activity by quantitative polymerase chain reaction, western blot analysis and enzymatic assays, respectively. Data are presented as the mean \pm standard error of the mean from three independent experiments. " $\mathrm{P}<0.05$ vs. control. NQO1, NAD(P)H:Quinone oxidoreductase 1 ; siRNA, small interfering RNA; NT, non-targeting siRNA; OD, optical density.

non-targeting siRNA-transfected control cells $(\mathrm{P}<0.05$; Fig. 2). These results suggested that knockdown of NQO1 transcripts using siRNA transfection decreases the ability of CCA cells to grow and proliferate, resulting in a decrease in colony formation. Therefore, these results implied that NQO1 may serve a role in CCA cell growth and proliferation.

Knockdown of NQO1 expression arrests the cell cycle progression of KKU-100 cells. To investigate the role of NQO1 on the regulation of the CCA cell cycle, NQO1-knockdown cells using siRNA were subjected to cell cycle analysis using a flow cytometer. The results demonstrated that NQO1-knockdown CCA cells significantly accumulated at the $\mathrm{G}_{1}$ phase with a decreased proportion of cells at the $S$ phase compared with the non-targeting siRNA-transfected control cells $(\mathrm{P}<0.05$; Fig. 3A). Therefore, these results suggested that NQO1 serves a role in cell cycle progression in CCA cells.
Knockdown of NQO1 expression contributes to altered levels of 21 and cyclin D1 protein. The suppression of cell proliferation and arrestment of cell cycle in KKU-100 cells by NQO1 siRNA transfection led to further experiments investigating the influence of NQO1 knockdown on the expression levels of proteins which are associated with cell proliferation and cell cycle. The effect of NQO1 siRNA on the expression levels of p21, cyclin A and cyclin D1 protein was observed by western blot analysis. Knockdown of NQO1 transcripts using siRNA transfection significantly increased p21 and decreased cyclin D1 protein expression levels compared with in the non-targeting siRNA-transfected control cells $(\mathrm{P}<0.05$; Fig. 3B); however, cyclin A protein expression levels remained the same. As p21 and cyclin D1 are important cell cycle regulator proteins for $G_{1}$ and $S$ phases, altered expression levels of p21 and cyclin D1 protein in NQO1-knockdown cells may induce CCA cells arrest at the $\mathrm{G}_{1}$ phase. These results provided 
evidence for the biological role of NQO1 in cell proliferation and cell cycle regulation of CCA cells.

Knockdown of NQO1 expression delays CCA cell migration. To investigate the role of NQO1 on CCA cell migration, wound healing and Transwell migration assays were performed. Knockdown of NQO1 transcripts using siRNA transfection efficiently delayed cell migration, and this suppressive effect was observed early at $6 \mathrm{~h}$ and continued until $72 \mathrm{~h}(\mathrm{P}<0.05$; Fig. 4A). To confirm the anti-migration effect of NQO1 siRNA, a Transwell migration assay was additionally performed. The results revealed that NQO1-knockdown CCA cells significantly lost their migration ability, as presented in Fig. 4B $(\mathrm{P}<0.05)$. The cell migration of NQO1-knockdown CCA cells decreased by $\sim 60 \%$ compared with the non-targeting siRNA-transfected control cells. Therefore, NQO1 siRNA exhibited anti-migration ability on CCA cells as observed using wound healing and Transwell migration assays, and it was concluded that NQO1 serves a role in the migration of CCA cells.

Knockdown of NQO1 expression contributes to altered $M M P / T I M P$ mRNA ratios. Further to demonstrating the biological role of NQO1 in CCA cell migration, the effects of NQO1 knockdown on expression levels of migration-associated genes were investigated using RT-qPCR. The RT-qPCR results revealed that knockdown of NQO1 transcripts using siRNA transfection did not alter expression levels of MMP2, MMP3 and MMP9, but tended to increase level of TIMP1 and significantly increased the expression level of TIMP2 mRNA compared with control cells (data not shown). The effects of NQO1 knockdown on the MMP/TIMP mRNA ratios, which are generally recognized as the index of the relative inhibition of MMP activity $(24,29)$, were analyzed. NQO1-knockdown CCA cells demonstrated a decreased MMP9/TIMP1 ratio compared with in the control cells $(\mathrm{P}<0.05$; Fig. 5); suggesting that NQO1 siRNA has an inhibitory effect on MMP activity. The suppression of MMP activity in NQO1-knockdown cells provided evidence of the biological role of NQO1 in the migration of CCA cells.

\section{Discussion}

The involvement of NQO1 in various types of cancer has previously been demonstrated (9-16). The upregulation of NQO1 in certain types of solid tumor, including CCA (17), was associated with poor prognosis, thus NQO1 targeting has therapeutic potential $(10,11)$. The present study investigated the biological role of NQO1 in cell proliferation, cell cycle and migration of CCA. The in vitro experiments revealed that knockdown of NQO1 expression compromised the proliferation and reproductive ability of cells, arrested cell cycle progression and inhibited the motile capacity of CCA cells. Therefore, suppression of NQO1 may be a potential target for the treatment and/or strategy to enhance the chemosensitivity of CCA and other types of cancer with increased NQO1 activity.

Previous studies have suggested possible growth-inhibitory effects triggered by NQO1 suppression $(11,18,19)$. Dicoumarol, a potent inhibitor of the NQO1 enzyme, inhibited cell growth
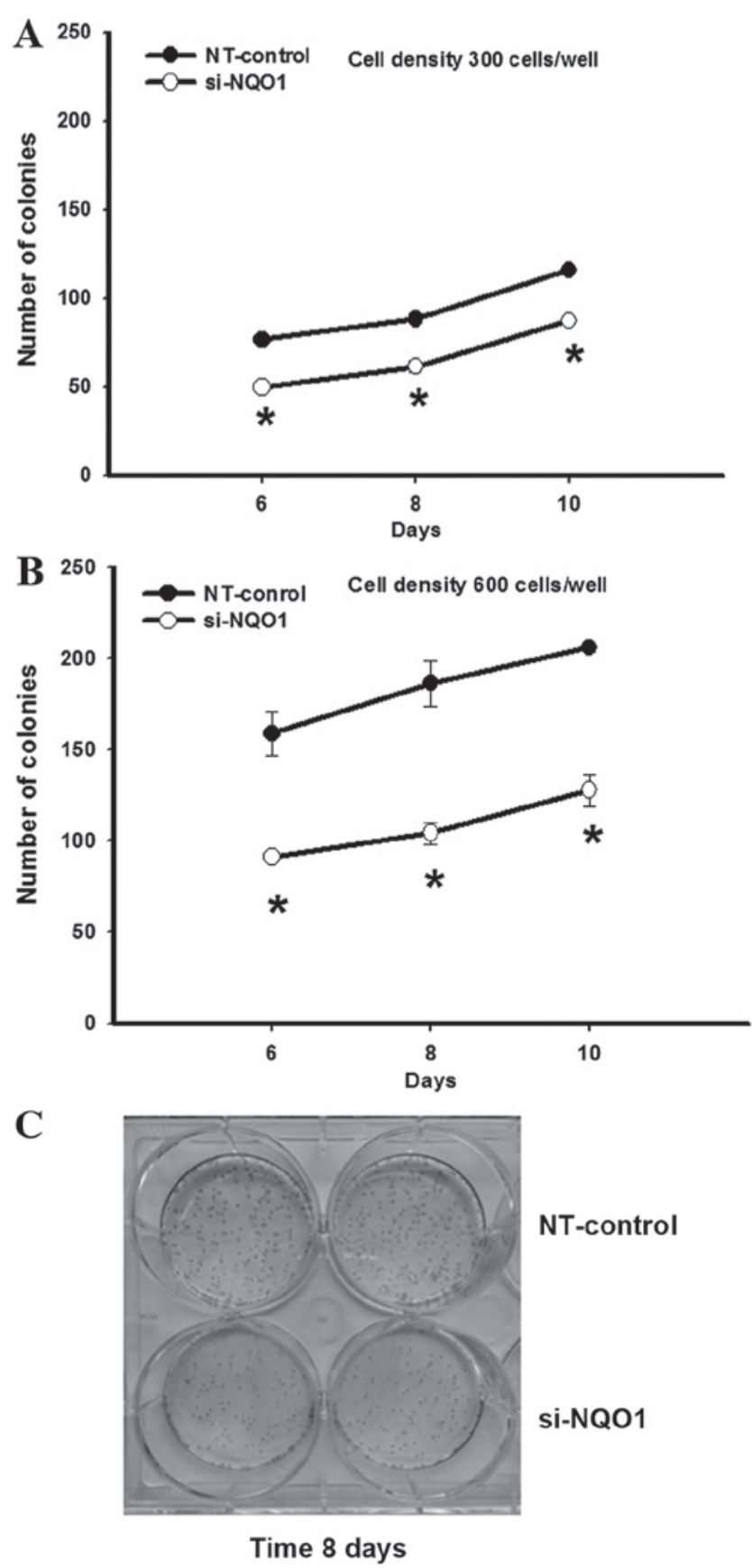

Figure 2. NQO1 siRNA reduced KKU-100 cell proliferation. NQO1-knockdown cells using siRNA were subjected to clonogenic survival assay. NQO1-knockdown CCA cells were plated at cell densities of (A) 300 and (B) 600 cells/well and cultured for another 10 days. The cell colonies were counted at 6,8 and 10 days. (C) Representative images of the clonogenic survival assay of the cell density at 600 cells/well cultured for 8 days. Data are presented as the mean \pm standard error of the mean from three independent experiments. " $\mathrm{P}<0.05$ vs. control. NQO1, $\mathrm{NAD}(\mathrm{P}) \mathrm{H}$ :quinone oxidoreductase 1; siRNA, small interfering RNA; NT, non-targeting siRNA.

of pancreatic cancer cells in a dose-dependent manner (30), whereas a higher dose of dicoumarol decreased colony formation on soft agar of pancreatic cancer cells (18). For HeLa cells, treatment with dicoumarol resulted in a marked decrease in the viability of cells and proliferation rate (28). In accordance with these findings, the results of the present study demonstrated that knockdown of NQO1 in CCA cells significantly impaired cell proliferative ability. These results 

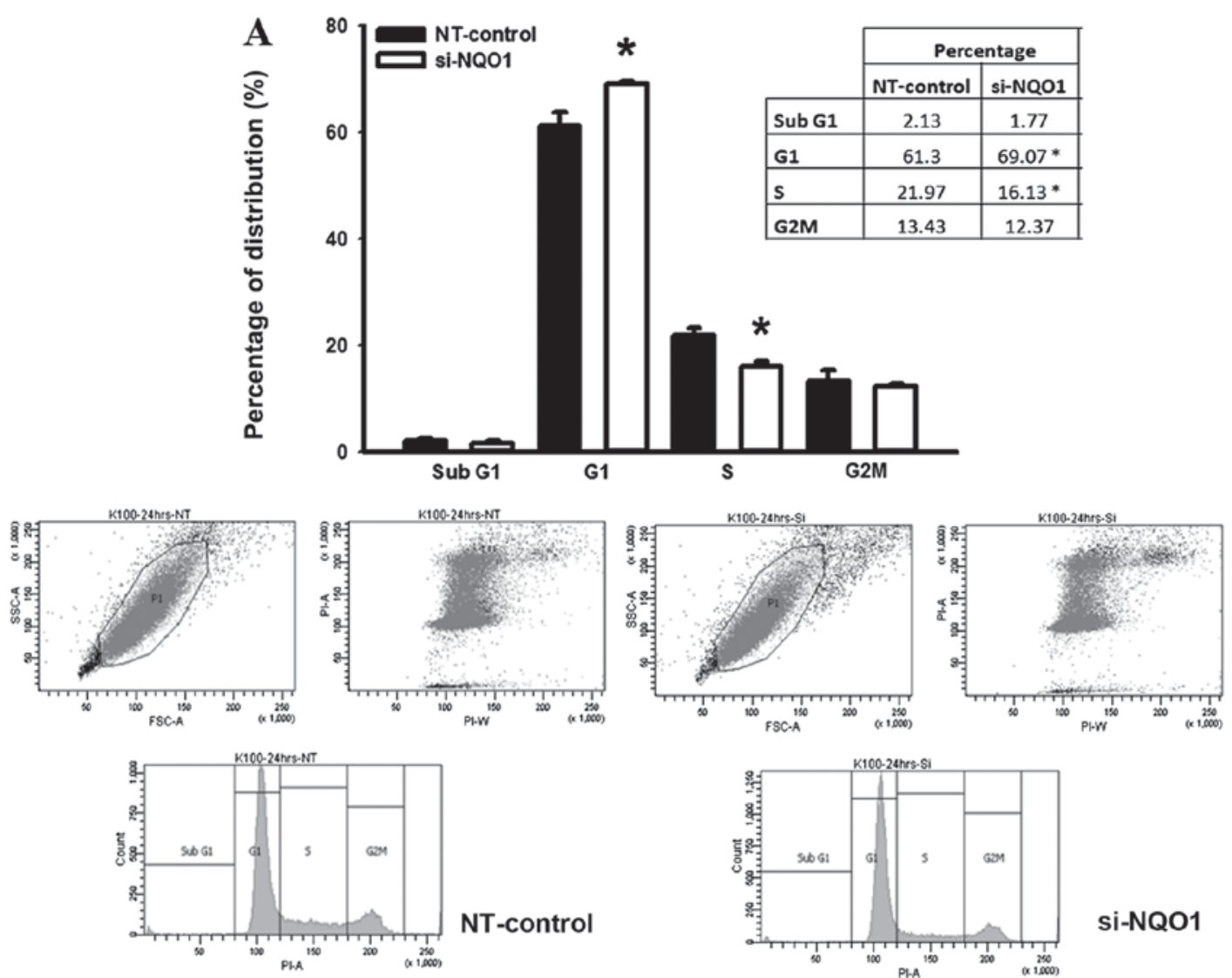

si-NQ01
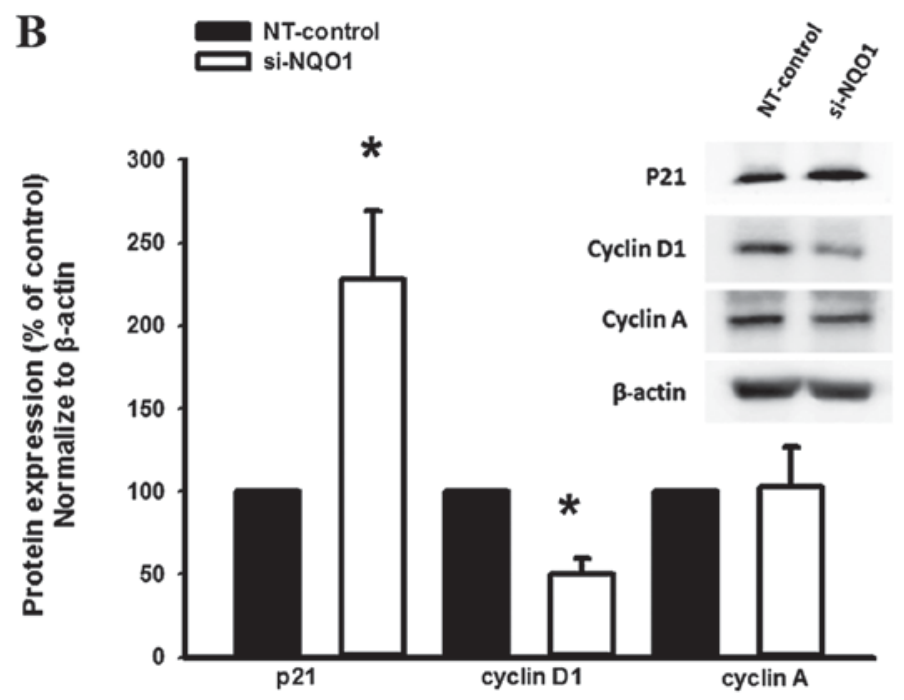

Figure 3. NQO1 siRNA (A) induced cell cycle arrest and (B) altered the expression of cell cycle and proliferation-associated proteins in KKU-100 cells. NQO1-knockdown cells using siRNA were synchronized by 12-h starvation, cultured for another $24 \mathrm{~h}$ and then subjected to flow cytometric analysis. (A) The cell cycle distribution at $24 \mathrm{~h}$ post-synchronization. (B) western blot analysis was performed using anti-p21, -cyclin D1, -cyclin A and $\beta$-actin antibodies. The expression levels of proteins were normalized using $\beta$-actin as a loading control for each band. Data are presented as the mean \pm standard error of the mean from three independent experiments. " $\mathrm{P}<0.05$ vs. control. NQO1, NAD(P)H:quinone oxidoreductase 1; siRNA, small interfering RNA; NT, non-targeting siRNA.

provide evidence suggesting the role of NQO1 in maintaining the ability of cell proliferation and replication.

The cell cycle, a series of events leading to cell division and duplication, is a key regulatory process in cell growth and proliferation (31). In the present study, flow cytometric analysis revealed that $\mathrm{NQO} 1$ knockdown induced $\mathrm{G}_{1}$ phase arrest and decreased the proportion of cells at the $\mathrm{S}$ phase, which may have contributed to the inhibition of proliferation in the NQO1-knockdown cells. In order to elucidate the possible molecular mechanism underlying NQO1-mediated inhibition of the proliferation and reproductive capacities of CCA cells, western blot analysis was performed to identify the influence of NQO1 siRNA on the expression levels of p21, cyclin A and cyclin D1 proteins. The results of the present study demonstrated that the upregulation of p21 and downregulation of cyclin D1 proteins were associated with the NQO1 expression 
$\mathbf{A}$
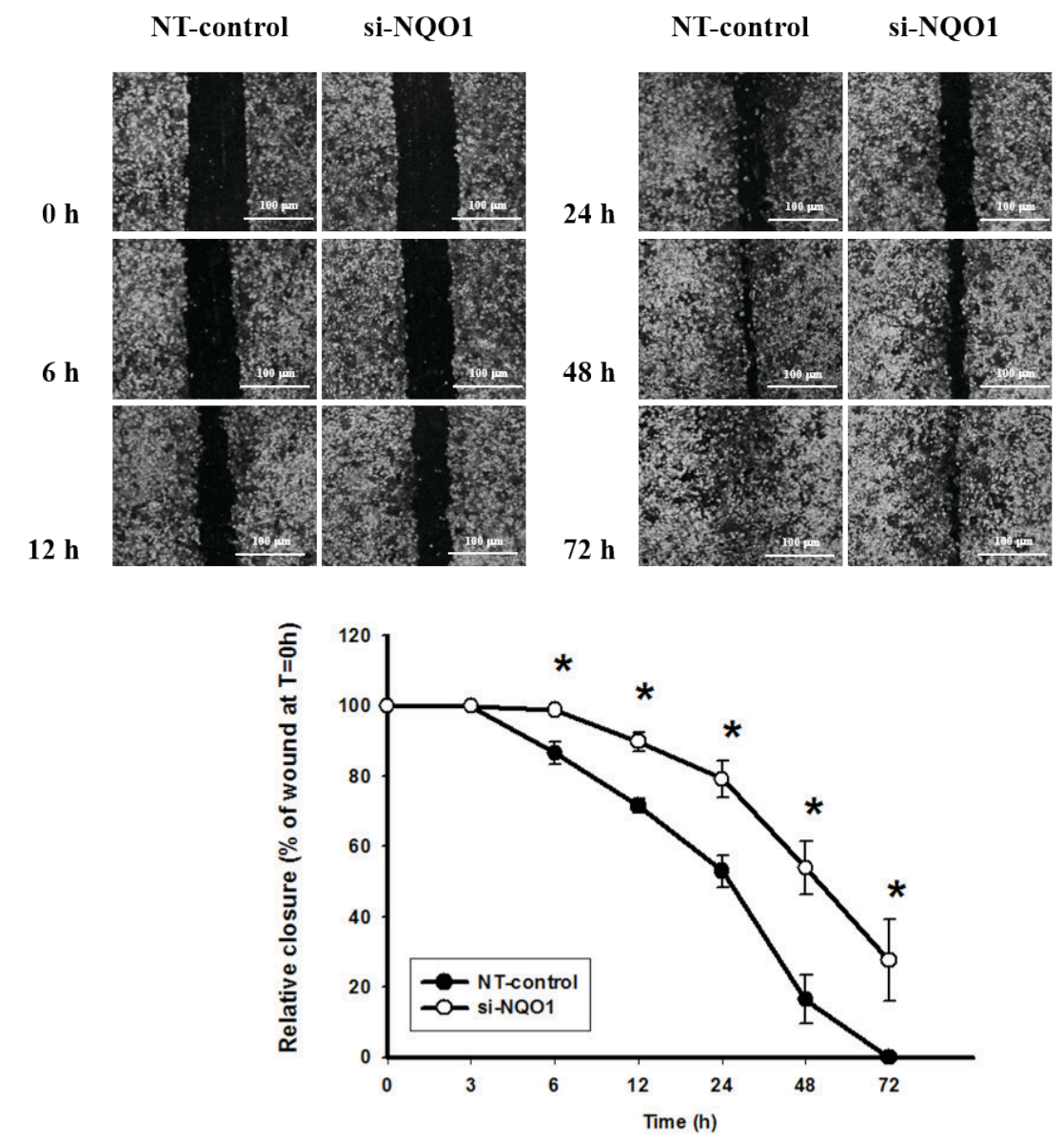

B

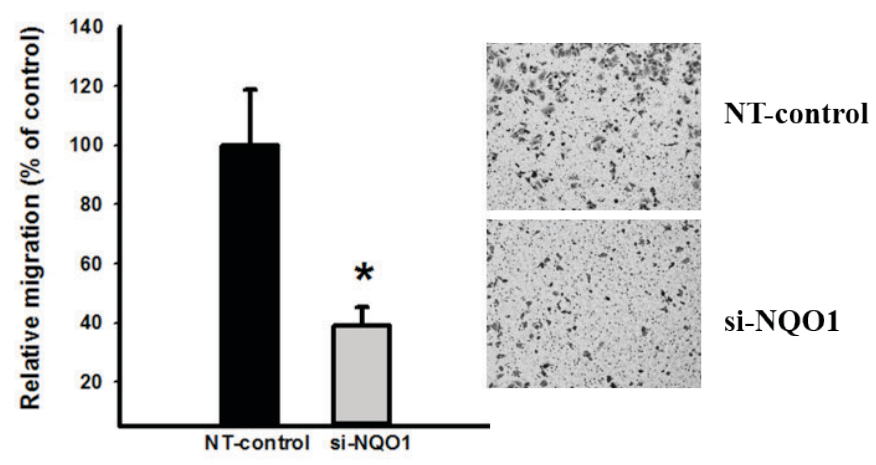

Figure 4. NQO1 siRNA delayed cell migration in KKU-100 cells. NQO1-knockdown cells using siRNA were subjected to (A) wound healing and (B) Transwell migration assays. (A) Representative images of the wound healing assays. The distance of cell migration into the scratched area was imaged ( $\mathrm{x} 4$ phase-contrast microscope) and measured at various times. The wound space was determined as the percentage of wound distance at initial time $(0 \mathrm{~h})$. (B) The Transwell migration assay was performed using modified Transwell chambers. Representative images of the Transwell migration assay. By systematical selection, 6 fields/well were imaged (x10 phase-contrast microscope) at $24 \mathrm{~h}$. The number of cells migrated to the lower surface was evaluated as a percentage of the control relative migration. The scale bar shows $100 \mu \mathrm{m}$. Data are presented as the mean \pm standard error of the mean from three independent experiments. "P<0.05 vs. control. NQO1, NAD(P)H:quinone oxidoreductase 1; siRNA, small interfering RNA; NT, non-targeting siRNA; T, time.

level, which indicated that these two proteins serve important roles in the process of antiproliferation and cell cycle arrest triggered by NQO1 siRNA. A similar observation was previously revealed in melanoma cells, where knockdown of NQO1 decelerated the transition of melanoma cells from $\mathrm{G}_{1}$ to $\mathrm{G}_{2}-\mathrm{M}$ phase (32). Knockdown of NQO1 in melanoma cells also induced downregulation of cyclins D1, A2 and B1 (32). Furthermore, treatment with dicoumarol increased the number of cells that accumulated in the sub- $\mathrm{G}_{1}$ phase (33).

Cell migration serves an important role in the cancer progression (34). The ability of NQO1 to drive human aortic vascular smooth muscle cell migration and invasion was 

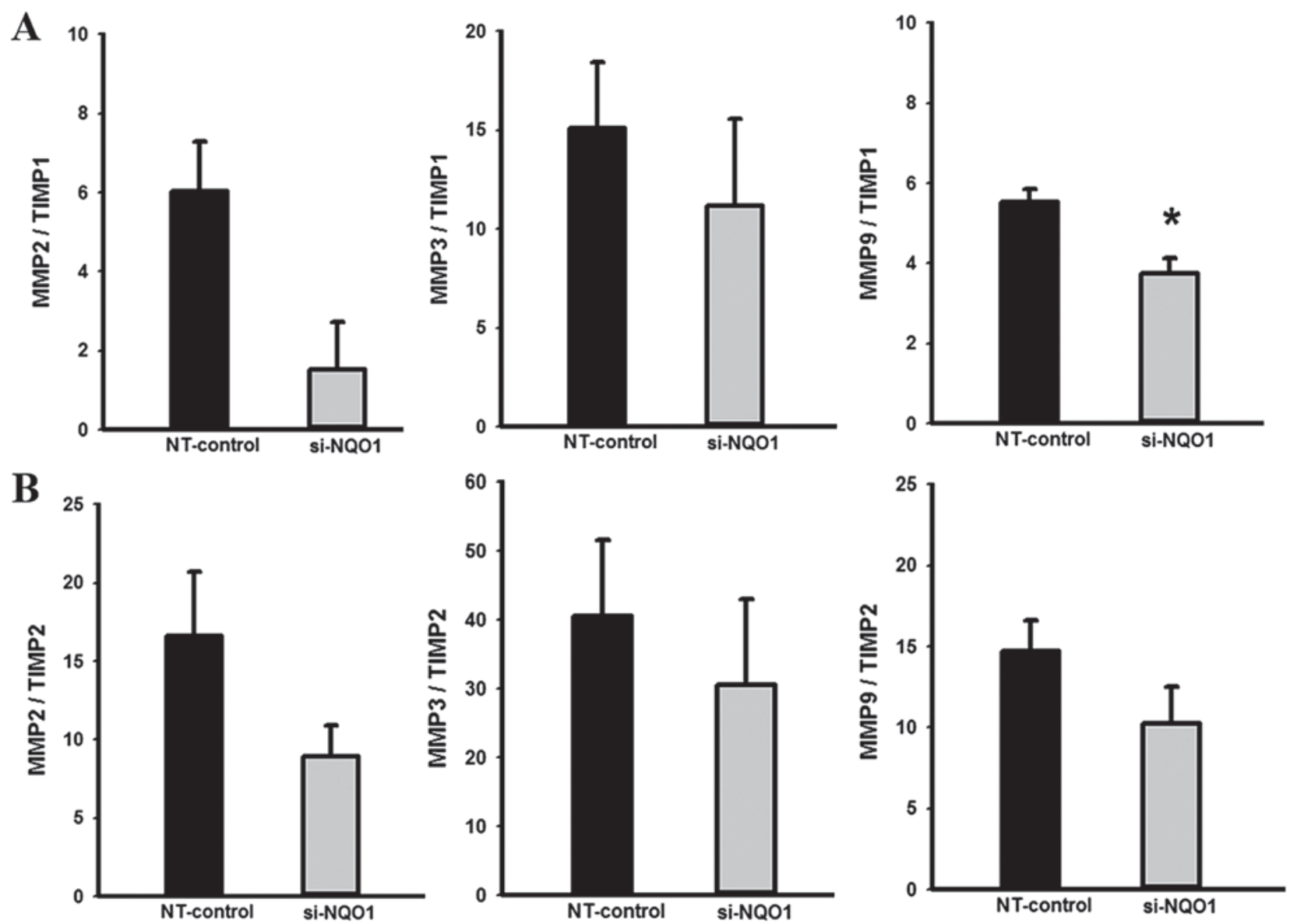

Figure 5. NQO1 siRNA decreased MMP/TIMP ratios. NQO1-knockdown cells using siRNA were subjected to RT-qPCR analysis. The mRNA levels of MMP2, MMP3, MMP9, TIMP1 and TIMP2 were normalized using $\beta$-actin mRNA as an internal control of each gene expression. The (A) MMP/TIMP1 and (B) MMP/TIMP2 mRNA ratios were determined in order to evaluate the relative inhibition of the MMP activity. Data are presented as the mean \pm standard error of the mean from three independent experiments. " $\mathrm{P}<0.05$ vs. control. RT-qPCR, reverse transcription-quantitative polymerase chain reaction; NQO1, NAD(P)H:quinone oxidoreductase 1; siRNA, small interfering RNA; MMP, matrix metalloproteinase; TIMP, tissue inhibitor of metalloproteinases; NT, non-targeting siRNA.

previously demonstrated (35). Treatment with dicoumarol or transfection with NQO1 siRNA suppressed tumor necrosis factor $\alpha$-induced cell migration by inhibiting MMP9 protein and mRNA expression $(35,36)$. In the present study, NQO1 siRNA significantly decreased the migratory ability of CCA cells, which suggested a role for NQO1 in promoting the metastasis of CCA. In order to elucidate the potential molecular mechanism underlying NQO1-mediated inhibition of the motility of the CCA cells, RT-qPCR analysis was performed to investigate the influence of NQO1 siRNA on the expression levels of MMP2, MMP3, MMP9, TIMP1 and TIMP2 mRNA. It was revealed that NQO1 siRNA exhibited an inhibitory effect on MMP activity by altering the MMP/TIMP mRNA ratio, which indicated that $\mathrm{NQO} 1$ serves a role in the process of cell migration of CCA.

In conclusion, to the best of our knowledge, the results of the present study provided evidence for the first time that NQO1 serves a key role in cell proliferation, cell cycle and migration of CCA cells. Furthermore, NQO1 siRNA demonstrated an antiproliferative effect in KKU-100 cells by upregulation of $\mathrm{p} 21$ and downregulation of cyclin D1 proteins. Additionally, NQO1 siRNA revealed an anti-migratory effect by decreasing the MMP9/TIMP1 mRNA ratio. Therefore, the results of the present study highlighted the biological role of NQO1 maintaining the ability of cell proliferation and replication and migration in CCA. Thus, NQO1 may be a potential molecular target to enhance CCA treatment and other types of cancer with increased NQO1 activity.

\section{Acknowledgements}

This present study was supported by Khon Kaen University Thailand Research Fund (grant no. RMU5380027), Faculty of Medicine (grant no. I57206) and a scholarship from the National Research University Project and Office of Higher Education Commission through the Specific Health Problem in Greater Mekong Sub-region project of Khon Kaen University to S.B.

\section{References}

1. Sripa B and Pairojkul C: Cholangiocarcinoma: Lessons from Thailand. Curr Opin Gastroenterol 24: 349-356, 2008.

2. Khan SA, Davidson BR, Goldin RD, Heaton N, Karani J, Pereira SP, Rosenberg WM, Tait P, Taylor-Robinson SD, Thillainayagam AV, et al: Guidelines for the diagnosis and treatment of cholangiocarcinoma: An update. Gut 61: 1657-1669, 2012. 
3. Anderson CD, Pinson CW, Berlin J and Chari RS: Diagnosis and treatment of cholangiocarcinoma. Oncologist 9: 43-57, 2004.

4. Patel T: Cholangiocarcinoma-controversies and challenges. Nat Rev Gastroenterol Hepatol 8: 189-200, 2011.

5. Buranrat B, Prawan A, Kukongviriyapan U, Kongpetch S and Kukongviriyapan V: Dicoumarol enhances gemcitabine-induced cytotoxicity in high NQO1-expressing cholangiocarcinoma cells World J Gastroenterol 16: 2362-2370, 2010.

6. Kongpetch S, Kukongviriyapan V, Prawan A, Senggunprai L, Kukongviriyapan $U$ and Buranrat B: Crucial role of heme oxygenase-1 on the sensitivity of cholangiocarcinoma cells to chemotherapeutic agents. PLoS One 7: e34994, 2012.

7. Samatiwat P, Prawan A, Senggunprai L and Kukongviriyapan V: Repression of Nrf2 enhances antitumor effect of 5-fluorouracil and gemcitabine on cholangiocarcinoma cells. Naunyn Schmiedebergs Arch Pharmacol 388: 601-612, 2015.

8. Zeekpudsa P, Kukongviriyapan V, Senggunprai L, Sripa B and Prawan A: Suppression of NAD $(\mathrm{P}) \mathrm{H}$-quinone oxidoreductase 1 enhanced the susceptibility of cholangiocarcinoma cells to chemotherapeutic agents. J Exp Clin Cancer Res 33: 11, 2014.

9. Ross D, Kepa JK, Winski SL, Beall HD, Anwar A and Siegel D $\mathrm{NAD}(\mathrm{P}) \mathrm{H}$ :Quinone oxidoreductase 1 (NQO1): Chemoprotection, bioactivation, gene regulation and genetic polymorphisms. Chem Biol Interact 129: 77-97, 2000.

10. Dinkova-Kostova AT and Talalay P: NAD(P)H:Quinone acceptor oxidoreductase 1 (NQO1), a multifunctional antioxidant enzyme and exceptionally versatile cytoprotector. Arch Biochem Biophys 501: 116-123, 2010.

11. Siegel D, Yan C and Ross D: NAD(P)H:Quinone oxidoreductase 1 (NQO1) in the sensitivity and resistance to antitumor quinones. Biochem Pharmacol 83: 1033-1040, 2012.

12. Basu S, Brown JE, Flannigan GM, Gill JH, Loadman PM, Martin SW, Naylor B, Puri R, Scally AJ, Seargent JM, et al: NAD(P)H:Quinone oxidoreductase-1 C609T polymorphism analysis in human superficial bladder cancers: Relationship of genotype status to NQO1 phenotype and clinical response to Mitomycin C. Int J Oncol 25: 921-927, 2004.

13. Cheng Y, Li J, Martinka M and Li G: The expression of NAD(P) $\mathrm{H}$ :Quinone oxidoreductase 1 is increased along with NF-kappaB p105/p50 in human cutaneous melanomas. Oncol Rep 23: 973-979, 2010

14. Cui X, Jin T, Wang X, Jin G, Li Z and Lin L: NAD(P)H:Quinone oxidoreductase-1 overexpression predicts poor prognosis in small cell lung cancer. Oncol Rep 32: 2589-2595, 2014

15. Kolesar JM, Pritchard SC, Kerr KM, Kim K, Nicolson MC and McLeod H: Evaluation of NQO1 gene expression and variant allele in human NSCLC tumors and matched normal lung tissue. Int J Oncol 21: 1119-1124, 2002.

16. Cui X, Li L, Yan G, Meng K, Lin Z, Nan Y, Jin G and Li C: High expression of NQO1 is associated with poor prognosis in serous ovarian carcinoma. BMC Cancer 15: 244, 2015.

17. Buranrat B, Chau-in S, Prawan A, Puapairoj A, Zeekpudsa P and Kukongviriyapan V: NQO1 expression correlates with cholangiocarcinoma prognosis. Asian Pac J Cancer Prev 13 (Suppl): S131-S136, 2012.

18. Cullen JJ, Hinkhouse MM, Grady M, Gaut AW, Liu J,Zhang YP, Weydert CJ, Domann FE and Oberley LW: Dicumarol inhibition of NADPH: Quinone oxidoreductase induces growth inhibition of pancreatic cancer via a superoxide-mediated mechanism. Cancer Res 63: 5513-5520, 2003.

19. Reigan P, Colucci MA, Siegel D, Chilloux A, Moody CJ and Ross D: Development of indolequinone mechanism-based inhibitors of NAD(P)H:Quinone oxidoreductase 1 (NQO1): NQO1 inhibition and growth inhibitory activity in human pancreatic MIA PaCa-2 cancer cells. Biochemistry 46: 5941-5950, 2007.
20. Matsui Y, Watanabe J, Ding S, Nishizawa K, Kajita Y, Ichioka K, Saito R, Kobayashi T, Ogawa O and Nishiyama H: Dicoumarol enhances doxorubicin-induced cytotoxicity in p53 wild-type urothelial cancer cells through p38 activation. BJU Int 105: 558-564, 2010

21. Wakai T, Shirai Y, Sakata J, Matsuda Y, Korita PV, Takamura M, Ajioka Y and Hatakeyama K: Prognostic significance of NQO1 expression in intrahepatic cholangiocarcinoma. Int J Clin Exp Pathol 4: 363-370, 2011

22. Sripa B, Leungwattanawanit S, Nitta T, Wongkham C, Bhudhisawasdi V, Puapairoj A, Sripa C and Miwa M: Establishment and characterization of an opisthorchiasis-associated cholangiocarcinoma cell line (KKU-100). World J Gastroenterol 11: 3392-3397, 2005.

23. Aneknan P, Kukongviriyapan V, Prawan A, Kongpetch S, Sripa B and Senggunprai L: Luteolin arrests cell cycling, induces apoptosis and inhibits the JAK/STAT3 pathway in human cholangiocarcinoma cells. Asian Pac J Cancer Prev 15: 5071-5076, 2014

24. Figueira RC, Gomes LR, Neto JS, Silva FC, Silva ID and Sogayar MC: Correlation between MMPs and their inhibitors in breast cancer tumor tissue specimens and in cell lines with different metastatic potential. BMC Cancer 9: 20, 2009.

25. Kim HA, Yeo Y, Kim WU and Kim S: Phase 2 enzyme inducer sulphoraphane blocks matrix metalloproteinase production in articular chondrocytes. Rheumatology (Oxford) 48: 932-938, 2009.

26. Onodera M, Zen Y, Harada K, Sato Y, Ikeda H, Itatsu K, Sato H, Ohta T, Asaka M and Nakanuma Y: Fascin is involved in tumor necrosis factor-alpha-dependent production of MMP9 in cholangiocarcinoma. Lab Invest 89: 1261-1274, 2009.

27. Pardo A, Gibson K, Cisneros J, Richards TJ, Yang Y, Becerril C, Yousem S, Herrera I, Ruiz V, Selman M and Kaminski N: Up-regulation and profibrotic role of osteopontin in human idiopathic pulmonary fibrosis. PLoS Med 2: e251, 2005.

28. Yang J, Zheng J, Wu L, Shi M, Zhang H, Wang X, Xia N, Wang D, Liu X, Yao L, et al: NDRG2 ameliorates hepatic fibrosis by inhibiting the TGF- $\beta 1 /$ Smad pathway and altering the MMP2/TIMP2 ratio in rats. PLoS One 6: e27710, 2011.

29. Groblewska M, Siewko M, Mroczko B and Szmitkowski M: The role of matrix metalloproteinases (MMPs) and their inhibitors (TIMPs) in the development of esophageal cancer. Folia Histochem Cytobiol 50: 12-19, 2012.

30. Lewis A, Ough M, Li L, Hinkhouse MM, Ritchie JM, Spitz DR and Cullen JJ: Treatment of pancreatic cancer cells with dicumarol induces cytotoxicity and oxidative stress. Clin Cancer Res 10: 4550-4558, 2004.

31. Alenzi FQ: Links between apoptosis, proliferation and the cell cycle. Br J Biomed Sci 61: 99-102, 2004.

32. Garate M, Wani AA and Li G: The NAD(P)H:Quinone Oxidoreductase 1 induces cell cycle progression and proliferation of melanoma cells. Free Radic Biol Med 48: 1601-1609, 2010.

33. Scott KA, Barnes J, Whitehead RC, Stratford IJ and Nolan KA: Inhibitors of NQO1: Identification of compounds more potent than dicoumarol without associated off-target effects. Biochem Pharmacol 81: 355-363, 2011

34. Golias CH, Charalabopoulos A and Charalabopoulos K: Cell proliferation and cell cycle control: A mini review. Int J Clin Pract 58: 1134-1141, 2004.

35. Lee SO, Chang YC, Whang $\mathrm{K}, \mathrm{Kim} \mathrm{CH}$ and Lee IS: Role of NAD(P)H:Quinone oxidoreductase 1 on tumor necrosis factor-alpha-induced migration of human vascular smooth muscle cells. Cardiovasc Res 76: 331-339, 2007.

36. Friedl $\mathrm{P}$ and Wolf K: Tumour-cell invasion and migration: Diversity and escape mechanisms. Nat Rev Cancer 3: 362-374, 2003. 